\title{
Topical issue on exotic matter in neutron stars
}

Published online: 29 March 2016 - (c) Società Italiana di Fisica / Springer-Verlag 2016

The recent measurement of two-solar-mass pulsars has initiated an intense discussion on its impact on our understanding of the high-density matter in the cores of neutron stars. A number of pressing issues prompted by recent astrophysical observations include in particular the presence of quark matter in massive stars, and whether the occurrence of phases of exotic hadronic matter, such as hyperons, is still possible or can be excluded. We collect contributions from leading experts in these fields, both from theory of dense matter as from groups providing recent observational astrophysical data and heavy-ion data. The possibility of pure quark stars, hybrid stars and the nature of the QCD phase transition are discussed and their observational signals delineated.

David Blaschke, Jürgen Schaffner-Bielich, and Hans-Josef Schulze 